\title{
Gestión administrativa educativa en la formación basada por competencias en estudiantes de Educación Superior Tecnológica
}

\author{
Educational administrative management in competency-based training in students of Higher Technological \\ Education
}

Gestão administrativa educacional na formação por competências em alunos do Ensino Superior Tecnológico

ARTíCULO DE INVESTIGACIÓN

\section{Teófilo Félix Valentín Melgarejo ${ }^{1}$ tevame39@gmail.com https://orcid.org/0000-0002-4063-5516}

\author{
Tito Armando Rivera Espinoza ${ }^{1}$ \\ centroazar@gmail.com \\ https://orcid.org/0000-0002-9212-9148
}

\author{
Yasminda Doris Paz Poma ${ }^{2}$ \\ yasmindapaz1@gmail.com \\ https://orcid.org/0000-0002-5451-5945
}

\section{Pablo Lolo Valentín Melgarejo \\ pl.valentin.m@gmail.com \\ https://orcid.org/0000-0003-3431-588X}

\section{Ulises Espinoza Apolinario ${ }^{1}$ \\ ulises2912@gmail.com \\ https://orcid.org/0000-0002-4636-0605}

${ }^{1}$ Universidad Nacional Daniel Alcides Carrión, Cerro de Pasco-Perú

${ }^{2}$ Instituto de Educación Superior Tecnológico Público “Daniel Alcides Carrión”, Los Olivos-Perú

Recibido 11 de mayo 2021 | Arbitrado y aceptado 9 de junio 2021 | Publicado en 01 julio 2021

\section{RESUMEN}

La investigación de gestión administrativa educativa en la formación basada por competencias en estudiantes del Instituto de Educación Superior Tecnológico Público "Daniel Alcides Carrión" - Yanahuanca - Pasco, tuvo como objetivo establecer la gestión administrativa educativa en la formación basada por competencias, para lo cual se tuvo que determinar los procesos, objetivos y funciones, para lograr estos objetivos se empleó el tipo de estudio básico, en los niveles descriptivo y explicativo; con el diseño pre experimental. Se usó el método científico, experimental de campo, documental y bibliográfico; y la muestra conformada por 78 estudiantes, obteniendo como resultado $-10,46$ y $-9,32$ según decisión estadística $\leq \mathrm{de}$ la prueba de McNemar, por lo que; la gestión administrativa educativa establece la formación basada por competencias en estudiantes del Instituto de Educación Superior Tecnológico Público "Daniel Alcides Carrión" - Yanahuanca - Pasco.

Palabras clave: Administración; Educación; Competencias; formación; gestión

\section{ABSTRACT}

The educational administrative management research in competencybased training in students of "Daniel Alcides Carrión" Public Technological Higher Education Institute - Yanahuanca - Pasco, aimed to establish educational administrative management in competency-based training, for which it was had to determine the processes, objectives and functions, to achieve these objectives the type of basic study was used, at the descriptive and explanatory levels; with the pre-experimental design. The scientific, experimental field, documentary and bibliographic method was used; and the sample made up of 78 students, obtaining as a result -10.46 and -9.32 according to statistical decision $\leq$ of the McNemar test, therefore; The educational administrative management establishes the training based on competencies in students of the Public Technological Higher Education Institute "Daniel Alcides Carrión" - Yanahuanca Pasco.

Key words: Administration; Education; Skills; Training; Management

\section{RESUMO}

A pesquisa de gestão administrativa educacional na formação por competências em alunos do Instituto de Ensino Superior Tecnológico Público "Daniel Alcides Carrión" - Yanahuanca - Pasco, teve como objetivo estabelecer a gestão administrativa educacional na formação por competências, para a qual se teve que determinar os processos, objetivos e funções, para atingir esses objetivos foi utilizado o tipo de estudo básico, nos níveis descritivo e explicativo; com o desenho pré-experimental. Utilizou-se o método científico, de campo experimental, documental e bibliográfico; e a amostra foi composta por 78 alunos, obtendo-se como resultado -10,46 e -9,32 conforme decisão estatística $\leq$ do teste de McNemar, portanto; A gestão administrativa educacional estabelece a formação baseada em competências em alunos do Instituto Superior de Educação Tecnológica Pública "Daniel Alcides Carrión" - Yanahuanca Pasco.

Palavras-chave: Administração; Educação; Habilidades; Treinamento; Gestão 


\section{INTRODUCCIÓN}

La educación superior en el sistema educativo peruano exige formar profesionales idóneos y competentes que estén acorde a la demanda del sector productivo nacional e internacional, por lo que; actualmente los Institutos de Educación Superior Tecnológicos, según Linares (2015), hace mención:

"Instituciones que ofrecen la
formación técnica, la profesional
técnica y la profesional a través de
un currículo por competencias que
prepare para una cultura productiva
con visión empresarial y capacidad
emprendedora y responda a las
demandas del sector productivo de la
Región o del país" (p. 11).

Que tienen la obligación en formar estudiantes según el enfoque por competencias y brindar una educación integral a través de saber, saber ser, saber hacer y el saber convivir, el cual consiste en desarrollar conocimientos, habilidades, actitudes $\mathrm{y}$ las relaciones intrapersonales que permita al estudiante integrarse al ámbito laboral y social, desempeñándose conforme a las exigencias del contexto. Puede llevarse a cabo desde cualquier modelo pedagógico existente que implique un compromiso de cambio en la mentalidad para la educación y el aprendizaje (Tobón, 2005).

Sin embargo, en los institutos de educación superior tecnológica todavía se sigue dando la enseñanza magistral o expositiva "actividad centrada en el profesor, en la que el estudiante es un agente pasivo que "recibe" las explicaciones del agente transmisor" (Sánchez-Carracedo y Barba, 2019, p. 87), donde la participación del estudiante es limitado para la elaboración y aplicación de instrumentos y recursos pedagógicos, Además; en la gestión administrativa educativa muy pocas veces lo priorizan a la formación basada por competencias y a la calidad de la educación que permitan la inserción de los egresados al mercado laboral, ya que como institución formadora debe encontrarse involucrada en los logros y desaciertos de la educación nacional, teniendo en cuenta los niveles de la gestión administrativa educativa: proceso, objetivos y la función; y su importancia de esto en la formación por competencias. Al respecto; surge la necesidad responder: ¿Cómo es la gestión administrativa educativa en la formación basada por competencias?, y ¿Cuáles son los procesos, objetivos y funciones de la gestión administrativa educativa para la formación basada por competencias en estudiantes del Instituto de Educación Superior Tecnológico Público "Daniel Alcides Carrión" Yanahuanca - Pasco?

Salinas (2012) en el trabajo de investigación "Importancia de la gestión administrativa en el desempeño de los docentes del colegio nacional experimental Ambato de la ciudad de Ambato provincia de Tungurahua en el año lectivo 2010 2011" entre sus conclusiones hace mención, que existe impacto de la gestión administrativa en el desempeño de los docentes; Tacuche (2018) en la tesis "Gestión administrativa y calidad de servicio de la Institución Educativa N 32282 "San Miguel", Lauricocha, 2018", en sus conclusiones determinó la relación entre la gestión administrativa y la calidad de servicio; y Carrasco (2002) desarrolló el estudio "Gestión educativa y calidad de formación profesional en la Facultad de educación de la UNSACA", en la que demostró que la gestión institucional ejerce una relación directa y positiva con la formación profesional de los estudiantes, además; tras su estudio, se determinó también la existencia de una relación directa entre la gestión administrativa y la calidad de formación profesional siendo un $81,8 \%$ el índice de correlación referida a un nivel alto y positivo.

Asimismo; sobre el aporte teórico de gestión administrativa educativa, Alvarado (1998) hace referencia al "conjunto de teorías, técnicas, 
principios y procedimientos aplicados al desarrollo del sistema educativo, a fin de lograr un óptimo rendimiento en beneficio de la comunidad que sirve" (P. 18), "orientadas hacia la consecución de ciertos objetivos que se desarrollan en las diversas áreas de actividad de la organización y en cuyo diseño y evaluación participan, en alguna medida, las personas encargadas de llevarlas a cabo" (García et al., 2018, p. 207), para lo cual; introduce elementos de orden y racionalidad a la educación formal haciéndola más potente y efectiva (Yépez, 2013); que se encuentra vinculada al quehacer educativo donde intervienen diversas disciplinas de las ciencias sociales que inciden directamente en el desarrollo de una institución y del país. En este sentido, la adecuada gestión educativa debe ser concebida como una herramienta para generar oportunidades educativas de calidad para todas las personas usuarias del sistema educativo (Miranda y Rosabal, 2018).

De modo que, la gestión administrativa educativa en las instituciones debe enfocarse en lograr los objetivos educacionales en función a los estudiantes enfocados por competencias, para lo cual se requiere que el responsable tenga habilidades de liderazgo para conducir y lograr estas metas en bien de la comunidad educativa en aspectos técnico y productivos, que permite tomar decisiones oportunas y pertinentes.

Mientras que la formación basada en competencias, apunta al logro de un desempeño complejo e idóneo, que incluye saber ser, saber hacer, saber conocer y saber convivir; y que estas deben ser combinaciones dinámicas de recursos personales, complejos sistemas de comprensión y acción donde se relacionen los saberes (Cuadra, et al., 2018,). Para ello, debe partirse de la necesidad de solucionar un problema de desempeño pedagógico (Aguiar y Rodríguez, 2018), que va permitir orientar la formación humana de forma integral y mediar el desarrollo, el aprendizaje y la construcción de las competencias en los estudiantes (Medina, 2010).
También; la formación basada por competencias se caracteriza por ser un proceso continuo, sistemático y basado en evidencias: el primero, permite el carácter continuo del proceso como prioridad y obligan a la recuperación de evidencias; la segunda, permite organizar de forma lógica en todas sus fases en donde la máxima preocupación es la objetividad y totalidad de los datos obteniendo información válida y fiable (Iglesias, 2017); y el proceso basado en evidencias, permite la relación de los niveles de la competencia con los contenidos e indicadores de logro y luego se articulan con los instrumentos, las evidencias del saber, saber hacer, saber ser (Huerta, 2018), que se encuentran íntimamente relacionados con lo cognoscitivo, afectivo y psicomotor (Cejas, et al., 2019).

Así que, la formación basada en competencias se orienta a la formación integral del estudiante, que implica el desarrollo de saberes técnicos y habilidades para la empleabilidad en los aspectos prácticos, que permita al profesional a desempeñarse exitosamente. Para lo cual es necesario plantear alternativas en la gestión administrativa educativa como en los procesos, objetivos y la función, para la eficiencia, eficacia y calidad de la formación por competencia en los niveles de productividad y competitividad ante las exigencias del contexto para resolver situaciones de la realidad de manera creativa, critica y reflexiva.

La investigación tuvo como objetivo establecer la gestión administrativa educativa con la formación basada por competencias; y de manera específica determinar los procesos, objetivos y funciones de la gestión administrativa educativa y su relación con la formación basada por competencias en los estudiantes del Instituto de Educación Superior Tecnológico Público "Daniel Alcides Carrión” Yanahuanca - Pasco. Por lo que; busca establecer la gestión administrativa educativa para coadyuvar a la formación basada por competencias, lo que permitirá determinar su aporte teórico, su aporte metodológico, los instrumentos y procedimientos, 
para elevar el nivel de aprendizaje a fin de responder a las diferentes demandas del mercado laboral en estudiantes de Institutos de Educación Superior Tecnológicas, dada la imperiosa necesidad de corregir las deficiencias de la gestión y formación de los estudiantes.

\section{MÉTODO}

La investigación fue de tipo básico con los niveles descriptivo y explicativo; por cuanto se trató de describir la gestión administrativa educativa con sus respectivas dimensiones y explicar la relación con la formación basada por competencia, y fue de diseño pre experimental, "es útil como un primer acercamiento al problema de investigación en la realidad" (Hernández, et al., 2014, p. 141). Se empleó el método científico, contrastando las ideas con los datos de la realidad, obtenidos en el proceso investigativo de manera rigurosa (Hernández, et al., 2018), en lo especifico se utilizó el método experimental de campo "procedimiento científico que permite inducir relaciones empíricas entre variables o comprobar la veracidad de una hipótesis, ley o modelo, por medio de un experimento controlado" (Baena, 2017, p. 40); el documental y bibliográfico, que consistió en tomar información para la construcción de los antecedentes de estudio, marco teórico y la estadística de las fuentes documentales de los estudiantes en tratamiento, y por último; el método estadístico, con el fin de recopilar, organizar, codificar, tabular, presentar, analizar e interpretar los datos obtenidos en la muestra de estudio durante y al final de la investigación a través del Excel y el SPSS.23.

La población estuvo conformada por 318 estudiantes del Instituto de Educación Superior Tecnológico Público "Daniel Alcides Carrión" - Yanahuanca - Pasco, y la muestra por 78 estudiantes del programa de estudios de enfermería técnica, de tipo no probabilística e intencional "los elementos son escogidos con base en criterios o juicios establecidos por el investigador" (Gallardo, 2017, p. 66); siendo el $24,52 \%$ de la población total, por lo que; cumple con los requisitos mínimos del tamaño de muestra (10\%) en el caso de una muestra no probabilística (Kerlinger, 1996). Se usó como instrumento las fichas bibliográficas, de citas, de resumen y de lectura; y como técnicas la documental, la codificación y la tabulación.

Asimismo, para la para la validez y el nivel de confiabilidad del instrumento se consideró los valores entre 0 y 1 . El resultado pudo evaluarse estadísticamente tabulado por Aiken. "Es precisamente esta posibilidad de evaluar su significación estadística lo que hace a este coeficiente uno de los más apropiados para estudiar este tipo de validez" (Gamarra, et al., 2015, p. 309), por medio de coeficiente de Alfa de Crombach ( $\alpha$ ), utilizando el SPSS. 23, en una muestra piloto de 8 integrantes, según fórmula:

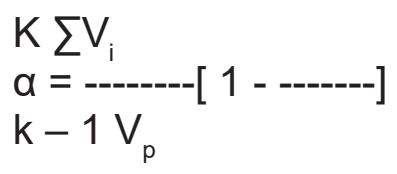

Donde:

a: coeficiente Alfa de Crombach

K: número de ítems en la prueba (18)

$\mathrm{V}_{\mathrm{i}}$ : varianza de cada ítem

$\mathrm{V}_{\mathrm{p}}$ : varianza de la prueba

Donde se obtuvo que $\alpha=0,76$; lo que permitió una confiabilidad alta por encontrarse en la escala $0,70 \leq a \leq 0,89$. Además; para establecer las inferencias estadísticas se eligió un nivel de significación de $5 \%(\alpha=0,05)$ y una aceptación de acierto al $95 \%$ por tratarse de una investigación educativo - social. 
RESULTADOS Y DISCUSIÓN

Se obtuvieron teniendo en cuenta el diseño de investigación pre experimental que consistió en la realización de una única medición temporal del fenómeno: gestión administrativa educativa para la formación basada por competencias. Estudio de caso con una sola medición, que consistió en administrar un estímulo o tratamiento a un grupo y después aplicar una medición a las variables para observar cuál es el nivel del grupo y luego realizar las comparaciones sobre los resultados.
Para el tratamiento y el resultado en la investigación se aplicó la prueba de McNemar "prueba no paramétrica de comparación de proporciones para dos muestras relacionadas" (Coronel-Carvajal, 2020, p. 5); obteniendo los datos producto de la observación de tipo cualitativo y aplicable a los diseños del tipo "antes y después" es decir en estudios longitudinales en los que cada elemento actúa como su propio control.

Tabla 1. Resultados de la gestión administrativa educativa.

\begin{tabular}{|c|c|c|c|c|c|c|}
\hline Dimensión & Condición & $\begin{array}{c}\text { Nunca } \\
(0)\end{array}$ & $\begin{array}{c}\text { Casi } \\
\text { nunca } \\
(1)\end{array}$ & $\begin{array}{c}\text { Indeciso } \\
\text { (2) }\end{array}$ & $\begin{array}{c}\text { Casi } \\
\text { siempre } \\
(3)\end{array}$ & $\begin{array}{c}\text { Siempre } \\
(4)\end{array}$ \\
\hline \multirow[t]{3}{*}{ Proceso } & Valoración en lo antes & 0,95 & 0,28 & 0,96 & 3,71 & 11,14 \\
\hline & Valoración en lo después & 6 & 4,11 & 1,07 & 0,45 & 0,41 \\
\hline & Tendencia diferenciada con respecto al después & $+5,05$ & $+3,83$ & $+0,11$ & $-3,26$ & $-10,73$ \\
\hline \multirow[t]{3}{*}{ Objetivos } & Valoración en lo antes & 1,13 & 0,35 & 1,13 & 3,9 & 13 \\
\hline & Valoración en lo después & 11,14 & 4,33 & 1,28 & 0,35 & 1 \\
\hline & Tendencia diferenciada con respecto al después & $+10,01$ & $+3,98$ & $+0,15$ & $-3,55$ & +12 \\
\hline \multirow[t]{3}{*}{ Función } & Valoración en lo antes & 1,04 & 0,21 & 0,98 & 3,90 & 11,14 \\
\hline & Valoración en lo después & 4,88 & 3,90 & 1,44 & 0,25 & 0,55 \\
\hline & Tendencia diferenciada con respecto al después & $+3,84$ & $+3,69$ & $+0,46$ & $-3,65$ & $-10,59$ \\
\hline
\end{tabular}

En la Tabla 1 se observa los resultados de las dimensiones de gestión administrativa educativa, las valoraciones en lo antes y después, y la tendencia diferenciada: en la dimensión de proceso se considera $+5,05$ Nunca, $+3,83$ Casi nunca, $+0,11$ Indeciso, -3,26 Casi siempre, y -10,73 Siempre, también; en la dimensión de objetivos se considera $+10,01$ Nunca, +3,98 Casi nunca, +0,15 Indeciso,
-3,55 Casi siempre, y +12 Siempre, asimismo; en la dimensión de función se considera $+3,84$ Nunca, $+3,69$ Casi nunca, +0,46 Indeciso, -3,65 Casi siempre, y -10,59 Siempre. Por lo que; según las tendencias diferenciadas acumuladas en la gestión administrativa educativa es a cero y se encuentra en el nivel alcanzado casi siempre y siempre (signo negativo), precisando el objetivo de estudio. 
Tabla 2. Diferencia de las dimensiones de la gestión administrativa educativa.

\begin{tabular}{cccccc}
\hline Dimensión & $\begin{array}{c}\text { Nunca } \\
(\mathbf{0})\end{array}$ & $\begin{array}{c}\text { Casi nunca } \\
(\mathbf{1})\end{array}$ & $\begin{array}{c}\text { Indeciso } \\
(\mathbf{2})\end{array}$ & $\begin{array}{c}\text { Casi siempre } \\
\mathbf{( 3 )}\end{array}$ & $\begin{array}{c}\text { Siempre } \\
\mathbf{( 4 )}\end{array}$ \\
\hline Proceso & $+5,05$ & $+3,83$ & $+0,11$ & $-3,26$ & $-10,73$ \\
Objetivos & $+10,01$ & $+3,98$ & $+0,15$ & $-3,55$ & +12 \\
Función & $+3,84$ & $+3,69$ & $+0,46$ & $-3,65$ & $-10,59$ \\
& $+18,90$ & $+11,50$ & $+0,72$ & $-10,46$ & $-9,32$ \\
Diferencia para McNemar & & $+31,12$ & & \multicolumn{2}{c}{$-19,78$} \\
& & & & & \\
\end{tabular}

En la Tabla 2 se observa los resultados según las diferencias para McNemar: $+18,90$ Nunca, $+11,50$ Casi nunca, +0,72 Indeciso, -10,46 Casi siempre, y -9,32 Siempre. Por lo que, considerando los dos últimos datos se observa una tendencia negativa e inclinación a cero como lo indica la teoría de la prueba de McNemar, además; como producto de la sumatoria de esta diferencia siendo $+31,12$ y $-19,78$ y de esta manera precisando el objetivo de estudio.

Tabla 3. Promedios de la formación basada por competencias.

\begin{tabular}{ccccc}
\hline Funtos & $\mathbf{f}_{\mathbf{i}}$ & $\mathbf{F}_{\mathbf{i}}$ & $\mathbf{h}_{\mathbf{i}}{ }$ & $\mathbf{H}_{\mathbf{i}} \mathbf{\%}$ \\
\hline$[10-20]$ & 1 & 1 & 1,28 & 1.28 \\
{$[30-40]$} & 1 & 2 & 1,28 & 2,56 \\
{$[50-60]$} & 10 & 12 & 12,82 & 15,38 \\
{$[70-80]$} & 37 & 49 & 47,44 & 62,82 \\
{$[90-100]$} & 29 & 78 & 37,18 & 100,0 \\
Total & $\mathbf{7 8}$ & & $\mathbf{1 0 0 , 0}$ & \\
\hline
\end{tabular}

La Tabla 3 muestra los promedios de las competencias investigativas, de los 78 estudiantes de la muestra de estudio, según parámetro planteado de 1 a 100 puntos, 37 de ellos se encuentran entre el intervalo 70 a 80 puntos y 29 de los mismos entre 90 a 100 puntos. Por lo que; en su representación porcentual mayoritaria se encuentran por encima de 70 puntos, con ello se afirma que la gestión administrativa educativa por medio de su: proceso, objetivos y función son óptimos para la formación basada por competencias y la relación que existe en entre ambas variables de estudio.

\section{Para la validación de los resultados se realizó} los siguientes procesos:

Consolidación del modelo 
Tabla 4. Determinación del modelo.

\begin{tabular}{cccc}
\hline & & \multicolumn{2}{c}{ Después } \\
\cline { 3 - 4 } & & + & - \\
Antes & + & $\mathrm{A}$ & $\mathrm{B}$ \\
& - & $\mathrm{C}$ & $\mathrm{D}$ \\
\hline
\end{tabular}

Determinación de la fórmula

$$
\chi_{\mathbb{N}}^{2}=\frac{(|A-D|-1)^{2}}{A+D}
$$

Dónde:

$\chi_{\mathbb{M}}^{2}=$ Valor estadística de McNemar

- $\mathrm{A}=$ Valor total de cambios observados en la medición después en casilla $\mathrm{A}$

- $\quad \mathrm{D}=$ Valor total de cambios observados en la medición después en casilla $\mathrm{D}$.
Aplicación de la prueba estadística: prueba de McNemar, es decir:

$$
X^{2}{ }_{M N}=\frac{(+/ 31,12--19,78 I-1)^{2}}{+31,12-19,78}=219,58
$$

Determinación del nivel de significación: valor de $\mathrm{p} \geq 0.05$

Cálculo de los grados de libertad (gl): siempre será igual a 1 , entonces $\chi_{(1) 0, \mathfrak{\theta})}^{2}=3,84$.

Regla de decisión: según la tabla de Chi-Cuadrada, se tiene que $\chi_{(1) 0, \mathbb{\theta})}^{2} \leq \chi_{\mathbb{M}}^{2}$

Tabla 5. Especificación y validación de los resultados.

\begin{tabular}{cccc}
\hline & & \multicolumn{2}{c}{ Después } \\
\cline { 3 - 4 } & & + & - \\
Antes & + & $+31,12$ & $-9,32$ \\
& - & $-10,46$ & $-19,78$ \\
\hline
\end{tabular}

\begin{tabular}{l|l|l|l} 
& & & \\
\hline & $-10,46$ & $-9,32$ & 0
\end{tabular}

Gráfico 1. Inclinación a cero. 
Por consiguiente, según los datos obtenidos, la gestión administrativa educativa establece la formación basada por competencias, tal como se observa en las diferencias de las dimensiones acumuladas de la gestión administrativa educativa (Tabla 2) donde el nivel alcanzado es "casi siempre" y "siempre" (signo negativo), también; la formación basada en competencias, en este caso los promedios de las competencias investigativas (Tabla 3) muestran que la mayoría de los estudiantes se encuentran por encima de 70 puntos, lo que sustenta los resultados de la decisión estadística $\chi_{(1) 0, \mathbb{\sigma})}^{2} \leq$ $\chi_{\mathbb{M}}^{2} ; 3,84 \leq 219,58$, y la prueba de McNemar donde los datos muestran $-10,46$ y $-9,32$ con tendencia negativa e inclinación a cero. Por lo que, la gestión administrativa educativa establece y determina la formación basada por competencias y la relación que existe entre ambas variables de estudio.

\section{Discusión}

Teniendo como referencia las investigaciones consideradas como antecedentes: Salinas (2012), entre sus conclusiones hace mención, que existe impacto de la gestión administrativa en el desempeño de los docentes; dicha referencia concuerda con los resultados obtenidos en la investigación, en este caso con respecto a los estudiantes, también; Tacuche (2018), entre sus conclusiones determinó la relación entre la gestión administrativa y la calidad de servicio, lo que; en la presente investigación concuerda con esa relación de la gestión administrativa educativa y la formación basada por competencias en los estudiantes; asimismo; Carrasco (2002) en su estudios que realizado determinó la existencia de una relación directa entre la gestión administrativa y la calidad de formación profesional siendo un $81,8 \%$ el índice de correlación referida a un nivel alto y positivo, situación que corrobora con lo demostrado en esta investigación, donde la gestión administrativa educativa establece la formación basada por competencias en los estudiantes del Instituto de Educación Superior Tecnológico Público "Daniel Alcides Carrión" - Yanahuanca - Pasco; obteniendo los datos -10,46 y -9,32 con una tendencia negativa e inclinación a cero como lo indica la teoría de la prueba de McNemar.

Las dimensiones de proceso, objetivos y de función de la gestión administrativa educativa, permitió obtener las diferencias según la prueba de McNemar, siendo +18,90 Nunca, +11,50 Casi nunca, +0,72 Indeciso, -10,46 Casi siempre, y -9,32 Siempre, permitiendo una tendencia positiva y negativa inclinándose a cero, y que la sumatoria de estas diferencias según los signos matemáticos generaron los resultados $+31,12$ y $-19,78$; lo mismo que sucedió con los promedios de los estudiantes en las formación competencias, en te caso investigativas la mayor cantidad se encontraron en el intervalo de 70 a 100 (66 estudiantes ) y solo (12 estudiantes) en el intervalo 0 a 60 , según parámetro planteado de 1 a 100 puntos; afirmándose que la gestión administrativa educativa por medio de sus dimensiones son óptimos para la formación basada por competencias, en los estudiantes del Instituto de Educación Superior Tecnológico Público "Daniel Alcides Carrión" - Yanahuanca - Pasco.

\section{CONCLUSIONES}

La gestión administrativa educativa establece y contribuye a la formación basada por competencias en estudiantes del Instituto de Educación Superior Tecnológico Público "Daniel Alcides Carrión” Yanahuanca - Pasco, tal como se demuestra en los resultados.

Las dimensiones de la gestión administrativa educativa: procesos, objetivos y funciones determinan en la formación basada por competencias y elevan el nivel de aprendizaje de los estudiantes del Instituto de Educación Superior Tecnológico Público "Daniel Alcides Carrión" Yanahuanca - Pasco. 


\section{REFERENCIAS}

Aguiar, X. M., y Rodríguez, L. (2018). La formación de competencias pedagógicas en los profesores universitarios. EDUMECENTRO, 10(2):141159. http://www.revedumecentro.sld.cu/index. $\mathrm{php} / \mathrm{edumc/article/view/1149}$

Alvarado, O. (1998). Gestión Educativa. Enfoques y Procesos. Editorial Fondo de Desarrollo Editorial.

Baena, G. (2017). Metodología de la investigación. Grupo Editorial Patria.

Carrasco, S. (2002). Gestión educativa y calidad de formación profesional en la facultad de educación de la UNSACA. [Tesis de maestría, Universidad Nacional Mayor de San Marcos]. https://cybertesis.unmsm.edu.pe/ handle/20.500.12672/1589

Cejas. M. F., Rueda, M. J., Cayo, L. E., y Villa, L. C. (2019). Formación por competencias: Reto de la educación superior. Revista de Ciencias Sociales, 25(1) https://www.redalyc.org/ journal/280/28059678009/html/

Coronel-Carvajal, C. (2020). Forma correcta de presentar los datos y uso de McNemar en las intervenciones educativas. Revista Archivo Médico de Camagüey, 24(1):4-7. http://www. revistaamc.sld.cu/index.php/amc/article/ view/6819/3477

Cuadra, D. J., Castro, P. J., y Juliá, M. T. (2018). Tres Saberes en la Formación Profesional por Competencias: Integración de Teorías Subjetivas, Profesionales y Científicas. Formación Universitaria, 11(5):19-30. http:// dx.doi.org/10.4067/S0718-50062018000500019

Gallardo, E. E. (2017). Metodología de la Investigación. Universidad Continental.

Gamarra, G., Rivera, T. A., Wong, F. J., y Pujay, O. E. (2015). Estadística e Investigación con aplicaciones de SPSS. Editorial San Marcos E. I. R. L

García, F. J., Juárez, S. C., y Salgado, L. (2018). Gestión escolar y calidad educativa. Revista Cubana Educación Superior, 37(2):206-216. http://scielo.sld.cu/pdf/rces/v37n2/rces16218. pdf
Hernández, R. Fernández, C., y Baptista, M. (2014). Metodología de la Investigación. Editorial McGraw-Hill

Hernández, A. A., Ramos, M. P., Placencia, B. M., Indacochea, B., Quimis, A. J., y Moreno, L. A. (2018). Metodología de la investigación científica. Editorial: Área de innovación y desarrollo, S. L.

Huerta, M. (2018). Evaluacion basada en evidencias, un nuevo enfoque de evaluación por competencias. Rev. Investig. Univ. Le Cordon Bleu, 5(1):159-171. https://doi.org/10.36955/ RIULCB.2018v5n1.0011

Iglesias, M. R. (2017). La evaluación basada en competencias. Monterrey.

Kerlinger, F. (1996); Investigación del comportamiento. Editorial McGraw-Hill

Linares, I. (2015). Situación de la educación superior tecnológica y técnico productiva hacia una política de calidad. Ministerio de Educación. https://www2.congreso.gob.pe/ sicr/cendocbib/con5_ https://www2.congreso. gob.pe/sicr/cendocbib/con5_uibd.nsf/ DAFD29C47494BD7005258312006FA34D/ \$FILE/SITUACION_DE_LA_EDUCACION_ SUPERIOR_TECNO.pdf

Miranda, L. A., y Rosabal, S. (2018). La gestión directiva en escuelas unidocentes y dirección 1: Un desafío para alcanzar la equidad educativa en contextos rurales de Costa Rica. Revista Electrónica Educare, 22(3):1-30. http://dx.doi. org/10.15359/ree.22-3.10

Medina, E. (2010). Formación integral y competencias. Pensamiento complejo, currículo, didáctica y evaluación. Revista Interamericana de Educación de Adultos, 32(2), 90-95. https:// www.redalyc.org/articulo.oa?id=457545095007

Salinas, S. E. (2012). Importancia de la gestión administrativa en el desempeño de los docentes del colegio nacional experimental Ambato de la ciudad de Ambato provincia de Tungurahua en el año lectivo 2010 - 2011. [Tesis de maestría, 
Universidad Técnica de Ambato]. https:// repositorio.uta.edu.ec/handle/123456789/2653

Sánchez-Carracedo, F., y Barba, A. (2019). Cómo impartir una clase magistral según la neurociencia. Actas de las Jenui, 4, 8794. https://upcommons.upc.edu/bitstream/ handle/2117/166394/478-3059-1 - PB. pdf? sequence $=1$

Tobón, S. (2005). Formación basada en competencias. Ecoe Ediciones

Tacuche, B. L. (2018). Gestión administrativa y calidad de servicio de la Institución Educativa $\mathrm{N}^{\circ} 32282$ “San Miguel”, Lauricocha, 2018. [Tesis de maestría, Universidad César Vallejo]. https://repositorio.ucv.edu.pe/ handle/20.500.12692/26432

Yépez, D. K. (2013). Modelo de gestión de orientación psicopedagógica como servicios educativos y vinculación con la comunidad de la Unidad Educativa Siete de Octubre, del Cantón Quevedo, Provincia de Los Ríos. [Tesis de maestria, Universidad Regional Autónoma de los Andes]. https://dspace.uniandes.edu.ec/ handle/123456789/3030

\section{Conflictos de intereses}

Los autores declaran que no existen conflictos de intereses.

\section{Contribución autoral}

Teófilo Félix Valentín Melgarejo: realizó la sistematización, redactó el artículo y corrigió el estilo según norma de redacción.

Yasminda Doris Paz Poma: realizó el trabajo metodológico, el recojo y la sistematización de datos.

Tito Armando Rivera Espinoza, realizó el procesamiento de los datos y apoyo en la sistematización de los resultados.

Pablo Lolo Valentín Melgarejo: realizó la búsqueda bibliográfica y redacto sobre formación basada en competencia.

Ulises Espinoza Apolinario, realizó la búsqueda bibliográfica y sobre la gestión administrativa educativa.

Además, todos los autores trabajaron de manera activa y consensuada el artículo. Por lo que; aprobamos para ser evaluados y su publicación del presente artículo. 